\title{
Características clínicas y enfoque terapéutico de pacientes con Malformaciones Anorrectales en dos Hospitales Sampedranos.
}

\author{
Clinical characteristics and therapeutic approach of patients with Anorrectal \\ Malformations in two Hospitals of San Pedro Sula.
}

\section{Gabriela Michelle Silva Zuniga*, Juan Craniotis Ríos**}

\section{RESUMEN}

Antecedentes: Las malformaciones anorrectales (MAR) se componen de una amplia gama de anormalidades que se asocian con varias patologías. Afectan tanto al sexo masculino como femenino, en algunos casos incluyen al aparato urinario y genital. Objetivo: Describir las características clínicas y el enfoque terapéutico de pacientes con Malformaciones Anorrectales en dos Hospitales de San Pedro Sula, Honduras, durante los años 2012 al 2016. Pacientes y Métodos: Estudio cuantitativo, descriptivo, transversal, realizado en pacientes con malformación anorrectal del Hospital Nacional Dr. Mario Catarino Rivas y el Hospital de Niños Quemados y Cirugía Pediátrica Ruth Paz. La muestra fueron 94 pacientes, la información se obtuvo del expediente clínico y se procesó en el software estadístico IBM SPSS 22. Resultados: En cuanto al sexo, $53.2 \%$ $(n=50)$ eran mujeres y $46.8 \%(n=44)$ hombres, $30.9 \%(n=29)$ tenían un año de edad, $51.1 \%$ $(n=48)$ provenían del departamento de Cortés. En los hombres, se encontró fístula perineal en $30.2 \%(n=14)$ pacientes $y$ en las mujeres se encontró fístula rectovestibular en $37.3 \%$ $(n=19)$, se encontró asociación a otro tipo de malformaciones congénitas en $29 \%(n=27)$ de los pacientes. El tratamiento consistió en colostomía protectora seguido de Anorrectoplastía sagital posterior tipo Peña y por último el programa de dilataciones anorrectalesa $59.6 \%(n=56)$ y $85.3 \%$ no presentó complica-

\footnotetext{
* Estudiante de Medicina de UNAH-VS

**Cirujano Pediatra, Director del Hospital para Niños Quemados y Cirugía Pediátrica Ruth Paz. (HPNQRP)

Dirigir correspondencia a: gabyssilva@hotmail.com

ciones quirúrgicas. Conclusiones: Las malformaciones anorrectales en este estudio afectaron más a las mujeres que a los hombres, la edad que predominó fueron los pacientes de 1 año, procedentes en su mayoría del departamento de Cortés, específicamente de San Pedro Sula.

\section{PALABRAS CLAVE}

Ano imperforado, Cloaca, Fístula, Colostomía.

\section{ABSTRACT}

Background: Anorectal malformations (MAR) are composed of a wide range of abnormalities that are associated with several pathologies, affecting both male and female sex, in some cases including the urinary and genital tract. Objective: To describe the clinical characteristics and therapeutic approach of patients with Anorectal Malformations in two hospitals in San Pedro Sula, Honduras, from the years 2012 untill 2016. Patients and Methods: A quantitative, descriptive, cross-sectional study of patients with anorectal malformations was conducted at the Dr. Mario Catarino Rivas National Hospital and the Ruth Paz Hospital of Burned Children and Pediatric Surgery. Results: In terms of sex, $53.2 \%(n=50)$ were women and $46.8 \%(n=44)$ men, 30.9\% $(n=29)$ were one year old, and $51.1 \%(n=48)$ came from the department of Cortes. Perineal fistula was found in $30.2 \%(n=14)$ male patients andrectovestibular fistula was found in $37.3 \%(n=19)$ females. Association with other types of congenital malformations was found in $29 \%(n=27)$ of the patients. The treatment consisted of a protective colostomy followed by a posterior 
Peña anorectoplasty and finally the program of anorectal dilatations to $59.6 \%$. $85.3 \%$ did not present surgical complications. Conclusions: Anorectal malformations in this study affected women more than men; the predominant age was 1 year-old patients, from the department of Cortes, specifically from San Pedro Sula.

\section{KEWORDS}

Anus Imperforate, Cloaca, Fistula, Colostomy.

\section{INTRODUCCIÓN}

Las malformaciones anorrectales (MAR) se componen de una amplia gama de anormalidades que se asocian con varias patologías, afectan tanto al sexo masculino como femenino, en algunos casos incluyen al aparato urinario y genital. ${ }^{(1,2)}$ Es necesario distinguir el tipo específico de MAR, ya que su adecuado diagnóstico y manejo terapéutico durante el período neonatal resultan determinantes en el futuro de un niño tanto desde el punto de vista funcional como psicosocial. Su incidencia ha sido estimada desde 1:1,500 hasta 1:5000 nacidos vivos. ${ }^{(3,4)}$

A nivel internacional se han realizado varios estudios sobre estos defectos congénitos. Sin embargo, en Honduras solo existe uno. Éste analiza la tasa de incidencia de Malformaciones Anorrectales (MAR) con 0.28 por cada 1000 nacidos vivos en la Unidad Materno Infantil del Hospital Escuela Universitario en Tegucigalpa. ${ }^{(5)}$ Por ser un tema poco conocido y estudiado en nuestro país, resultó interesante investigarlo, ya que aunque se conoce desde la antigüedad, sus enfoques terapéuticos y abordajes quirúrgicos han sido propuestos recientemente durante el siglo XX-XXI. ${ }^{(6)}$

Los factores que contribuyen a una buena calidad de vida de los pacientes con anomalías anorrectales, es el diagnóstico temprano y la cirugía exitosa llevada a cabo por profesionales expertos en estas malformaciones. La inconti- nencia fecal y urinaria puede ocurrir incluso luego de un abordaje quirúrgico exitoso, ya que en algunos casos existen otros problemas asociados en los pacientes como por ejemplo hipodesarrollo del sacro y anomalías de la médula espinal. ${ }^{(7,8)}$

El objetivo del estudio fue describir las características clínicas y el enfoque terapéutico de pacientes con Malformaciones Anorrectales en dos Hospitales de San Pedro Sula durante los años 2012 al 2016.

\section{MATERIALES Y METODOS}

Se realizó un estudio descriptivo, transversal. El universo fue de 105 pacientes con Malformación Anorrectal atendidos en dos hospitales: Hospital Nacional Dr. Mario Catarino Rivas (HNMCR) y Hospital para Niños Quemados y Cirugía Pediátrica Ruth Paz (HNQCPRP), durante enero del 2012 a diciembre del 2016. La muestra fue de 94 pacientes (86 pacientes del HNQCPRP y 8 pacientes del HNMCR) que cumplían con los criterios de inclusión: Pacientes con Malformación Anorrectal que nacieron o acudieron a algunos de los dos hospitales, en el período descrito, con expediente completo, los criterios de exclusión: pacientes con Malformación Anorrectal que nacieron en período de tiempo diferente o que fueron atendidos en otro hospital, pacientes con expediente incompleto. Los 94 pacientes proporcionan un intervalo de confianza de \pm 3.29 con un nivel de confianza del $95 \%$ y nivel de precisión de 50\%.

Los pacientes fueron captados en la consulta externa de cirugía pediátrica de ambos hospitales. Los pacientes al ingresar al estudio estaban en distintas fases de tratamiento. Los datos obtenidos de los expedientes clínicos se consignaron en un cuestionario de recolección que incluía sexo, edad, departamento, municipio, tipo de Malformación Anorrectal según sexo, asociación de MAR a otras malformaciones congénitas, tratamiento quirúrgico, complica- 
ciones quirúrgicas y complicaciones de colostomía; posteriormente se transcribió en una base de datos en IBM SPSS Versión 22, con la que fueron analizados. Para la presentación de datos se utilizó la hoja de Microsoft Excel 2010.

\section{RESULTADOS}

Se encontró que de los 94 pacientes estudiados el $53.2 \%(n=50)$ eran mujeres y $46.8 \%(n=44)$ eran hombres. El 51.1\% $(n=48)$ provenían del departamento de Cortés, $10.6(n=10)$ de Yoro, 8.5\% $(n=8)$ de Santa Bárbara, 7.4\% $(n=7)$ Atlántida, 5.3\% ( $n=5)$ Lempira, 4.3\% ( $n=4)$ Ocotepeque y Copán, 3.2\% (n=3) Francisco Morazán, 1.1\% ( $n=1)$ Intibucá, La Paz, Olancho, Colón y Comayagua. Del departamento de Cortés específicamente de la ciudad de San Pedro Sula
56.3\% ( $n=28), 18.8 \%(n=8)$ de Choloma, 8.3\% $(\mathrm{n}=4)$ Villanueva, $6.3 \%(\mathrm{n}=3)$ de La Lima y Omoa, $2.1 \%(n=1)$ de Pimienta y Puerto Cortés.

Dentro de los pacientes estudiados, se encontró que la edad mínima fue de 1 mes, la edad máxima 27 años, la media era 13 años y medio; y la moda 1 año de edad.

El tipo de Malformación Anorrectal que se encontró en el sexo masculino fue la fístula perineal en un $30.2 \%$ (14) pacientes, a diferencia del sexo femenino en donde la fístula rectovestibular se encontró en el 37.3\% (19), (Ver tabla No. 1). Se encontraron dos casos con síndrome de Down, los que presentaban agenesia anorrectal sin fístula.

Tabla No. 1: Tipo de Malformación Anorrectal según sexo.

\begin{tabular}{|c|c|c|c|}
\hline Sexo Masculino & n (\%) & Sexo Femenino & n (\%) \\
\hline Fístula Recto-Perineal & $14(30.2 \%)$ & Fístula Recto-Vestibular & $19(37.3 \%)$ \\
\hline Fístula Recto-Uretra-Vulvar & $8(18.6 \%)$ & Cloaca con canal común $>3 \mathrm{cms}$ & $8(15.7 \%)$ \\
\hline Estenosis Rectal & $7(16.3 \%)$ & Cloaca con canal común $<3 \mathrm{cms}$ & $7(13.7 \%)$ \\
\hline Atresia Rectal & $7(16.3 \%)$ & Estenosis Rectal & $6(11.8 \%)$ \\
\hline Fístula Recto-Uretra-Prostática & $5(11.6 \%)$ & Fístula Recto-Perineal & $5(9.8 \%)$ \\
\hline Fístula Recto-Uretra-Vesical & $2(4.7 \%)$ & Atresia Rectal & $3(5.9 \%)$ \\
\hline \multicolumn{2}{|c|}{ Fístula Recto-Uretral no especificado 1 (2.3\%) } & Fístula Recto-Vaginal & $2(3.9 \%)$ \\
\hline TOTAL & & $50(100 \%)$ & \\
\hline
\end{tabular}

Fuente: Investigación Características Clínicas y Enfoque Terapeútico en pacientes con MAR en dos Hospitales de SPS.

El 29\% $(n=27)$ de los casos en los que se encontró malformaciones asociadas; en $51.9 \%(n=14)$ había más de una malformación: Ausencia de sacro y cóccix $(n=2)$, ausencia de cóccix + displasia quística renal izquierda $(n=1)$, duplicación vaginal + duplicación Intestinal (recto) $(n=1)$, duplicación vaginal y cervical + vejiga trabeculada + ureter derecho doble $(n=1)$, hemivértebra + ausencia de sacro y cóccix + escoliosis lumbar + displasia de cadera $(n=1)$, Síndrome de Down + labio paladar hendido $(n=1)$, mielomeningocele + duplicación peneana y uretral $(n=1)$, 
Síndrome de Down + polidactilia+ criptorquidia $(n=1)$, ausencia de metatarsianos en ambos pies + ausencia de cúbito y radio en ambos brazos $(n=1)$, hidronefrosis + agenesia renal unilateral $(n=1)$, pie equinovaro izquierdo + polidactilia $(n=1)$, reflujo ureteral + hidronefrosis+ hipotrofia renal derecha + polidactilia $(n=1)$, reflujo ureteral + hidronefrosis+ displasia renal derecha + duplicación vaginal $(n=1)$. El $18.5 \%(n=5)$ bajo el ítem otro, corresponden a los pacientes que presentaron asociación al Síndrome de Down $(n=2)$, hipertrofia de labios menores $(n=1)$, ectopia renal $(n=1)$ y sacro fusionado $(n=1)$.

Mientras que la hidronefrosis se observó en el $7.4 \%(n=2)$, atresia esofágica $7.4 \%(n=2)$, reflujo ureteral $3.7 \%(n=1)$ respectivamente. (Ver gráfica No. 1).

\section{Gráfica No. 1: Tipo de malformación congé-} nita asociada.

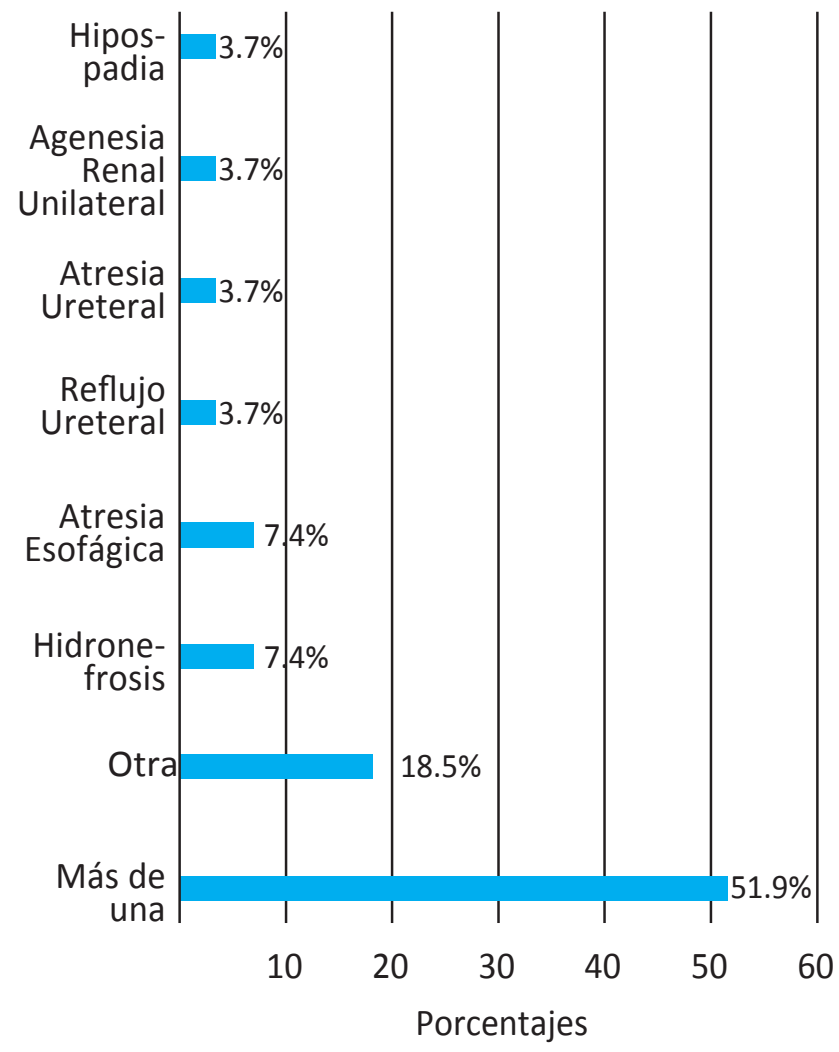

Fuente: Investigación Características Clínicas y Enfoque Terapeútico en pacientes con MAR en dos Hospitales de SPS.
En la tabla No. 2 se presenta la frecuencia y porcentaje de los tipos de tratamiento utilizados en los pacientes con MAR. Se encontró que el enfoque terapéutico utilizado en los pacientes con MAR es la Colostomía + Anorrectoplastía Sagital Posterior tipo Peña (ARPSP) y posteriormente se encuentran en el programa de dilataciones anorrectales a 59.6\% ( $n=56)$. Mientras que el $6.4 \%(n=6)$ de la opción otro corresponde a dilataciones anorrectales $(n=3)$, ARPSP + vaginoplastia $(n=1)$, Anoplastía Tipo Cutback $(n=1)$, Transposición anal Tipo Gross $(n=1)$.

\section{Tabla No. 2: Tipo de tratamiento utilizado en pacientes MAR.}

\begin{tabular}{lcc}
\hline \hline Tipo de Tratamiento Utilizado & $\mathrm{n}=94$ & $\%$ \\
\hline $\begin{array}{l}\text { Colostomía+ARPSP + } \\
\text { Dilataciones Anorrectales }\end{array}$ & 56 & 59.6 \\
$\begin{array}{l}\text { Colostomía sin } \\
\text { Reparación Anorrectal }\end{array}$ & 10 & 10.6 \\
$\begin{array}{l}\text { ARPSP* sin Colostomía+ } \\
\text { Dilataciones Anorrectales }\end{array}$ & 9 & 9.6 \\
$\begin{array}{l}\text { Otra } \\
\begin{array}{l}\text { Colostomía + Dilataciones } \\
\text { Anorrectales }\end{array}\end{array}$ & 6 & 6.4 \\
$\begin{array}{l}\text { Ano-Recto-Uretro-Vaginoplastía } \\
\text { con reemplazo Vaginal total }\end{array}$ & 5 & 5.3 \\
$\begin{array}{l}\text { Colostomía+ Anorrectoplastía } \\
\text { Sagital Posterior }\end{array}$ & 2 & 2.1 \\
$\begin{array}{l}\text { ARSP con Movilización } \\
\text { Urogenital Total }\end{array}$ & 1 & 1.1 \\
\hline
\end{tabular}

*ARPSP: Anorrectoplastía sagital posterior

Fuente: Investigación Características Clínicas y Enfoque

Terapeútico en pacientes con MAR en dos Hospitales de SPS. 
En la tabla No. 3 se presenta el tipo de complicación quirúrgica secundaria a colostomía y a reparación anorrectal. En total, se les realizó colostomía al $86 \%(n=81)$ de los pacientes, en el $89 \%(n=72)$ no presentaron complicación postquirúrgica, sin embargo $11.1 \% \quad(n=9)$ presentaron complicación, dentro de ellas la estenosis en $2.5 \% \quad(n=2)$, infección $(n=1)$ y hemorragia $(n=1)$. Se realizó la reparación anorrectal a $79.7 \%$ (75) de los casos, $85.3 \%(n=64)$ no presentaron complicación quirúrgica, sin embargo $14.7 \%(n=11)$ pacientes si presentaron complicaciones. La opción a más de una complicación secundaria a colostomía corresponde a obstrucción intestinal + peritonitis, mientras que en la segunda columna relacionada a reparación anorrectal corresponde a malposición de ano en previa ARPSP + dehiscencia de anoplastía; ambas ocurridas en el mismo paciente $(n=1)$.

Tabla No. 3: Tipo de complicación quirúrgica secundaria a colostomía y a reparación anorrectal.

\begin{tabular}{lcc}
\hline $\begin{array}{l}\text { Complicación } \\
\text { quirúrgica } \\
\text { secundaria a: }\end{array}$ & $\begin{array}{c}\text { Colostomía } \\
\mathrm{N}=81(\%)\end{array}$ & $\begin{array}{c}\text { Reparación } \\
\text { anorrectal } \\
\mathrm{n}=75(\%)\end{array}$ \\
\hline Ninguna & $89 \%(72)$ & $85.3 \%(64)$ \\
Estenosis & $2.5 \%(2)$ & $4 \%(3)$ \\
Prolapso rectal & $2.5 \%(2)$ & $2.7 \%(2)$ \\
Infección de la herida & $1.2 \%(1)$ & $1.3 \%(1)$ \\
Hemorragia & $1.2 \%(1)$ & $1.3 \%(1)$ \\
Hernia & $1.2 \%(1)$ & $0 \%(0)$ \\
Fístula recurrente & $0 \%(0)$ & $1.3 \%(1)$ \\
Obstrucción Intestinal & $1.2 \%(1)$ & $0 \%(0)$ \\
Más de una complicación & $1.2 \%(1)$ & $1.3 \%(1)$ \\
Dehiscencia de anoplastía & $0 \%(0)$ & $2.7 \%(2)$ \\
\hline
\end{tabular}

Fuente: Investigación Características Clínicas y Enfoque Terapeútico en pacientes con MAR en dos Hospitales de SPS.

\section{DISCUSION}

En este estudio realizado en ambos hospitales de la ciudad de San Pedro Sula, se encontró que las Malformaciones Anorrectales afectaron más a las mujeres $53.2 \%(n=50)$ que a los hombres $46.8 \%(n=44)$, mientras que el tipo de malformación anorrectal predominante en el sexo masculino fue la fístula recto-perineal $30.2 \%(n=14)$, a diferencia del sexo femenino en donde predominó la fístula rectovestibular 37.3\% $(n=19)$. La estadística sobre las características de la población con malformaciones anorrectales es variable de acuerdo al país donde se realiza, ya que en Cuba en donde se estudió a 55 recién nacidos intervenidos quirúrgicamente por malformaciones digestivas, atendidos en el Hospital Infantil Norte "Dr. Juan de la Cruz Martínez Maceira" predominó el sexo masculino $65.5 \%$. $(n=9)$, similar a los resultados de un estudio de 3 años en México con 72 casos, donde predominó el sexo masculino $58.3 \%(n=42)$ de los casos y la fístula recto uretral bulbar fue la MAR más común. ${ }^{(9,10)}$

Mientras que en Costa Rica en un análisis de 200 casos pediátricos de malformaciones anorrectales en el Hospital Nacional de Niños "Dr. Carlos Sáenz Herrera" se obtuvieron 92 casos de fístula perineal, 26 fístulas vestibulares y 12 fístulas recto-cuello-vesical. ${ }^{(11)}$

La edad de los pacientes en ambos Hospitales Sampedranos es variable, ya que son referidos de diversos centros asistenciales del país, es por éste motivo que ningún paciente es menor de un mes y están siendo manejados de acuerdo a su tipo de MAR y fase del tratamiento de forma ambulatoria mediante consulta externa.

Se presume que su etiología es multifactorial con una interacción causal de factores genéticos y ambientales, como por ejemplo: antecedentes familiares de primer y segundo grado con MAR, índice de masa corporal de la madre antes del embarazo $\geq 25 \mathrm{~kg} / \mathrm{m} 2$, fiebre durante 
el primer trimestre del embarazo, exposición materna laboral a agentes de limpieza y solventes, exposición paterna al tabaquismo y exposición laboral a gases de escape. ${ }^{(12)}$

En la presente investigación hubo asociación a malformaciones acompañantes en un $29 \%$ $(n=27)$, cifra que difiere con la literatura internacional por ejemplo en Chile en un estudio malformaciones congénitas, donde de 54 casos con MAR, el 51.9\% $(n=28)$ tenía asociación a otras anomalías. ${ }^{(13)}$

Otro dato de interés es que en los casos con síndrome de Down ( $n=4)$ se asociaron a agenesia anorrectal sin fístula, tal y como lo muestra el estudio retrospectivo de 34 años (19802014) hecho por el Dr. Peña, Bischoff, Frischer y Dickie quienes revisaron los expedientes clínicos de 2,189 pacientes encontrándose con 92 casos de Agenesia Anorrectal sin fístula y una asociación del $40 \%$ a Síndrome de Down. ${ }^{(14)}$ Muy similar al estudio realizado por Fanjul M. Molina E. Cerda J. en el año 2009 quienes estudiaron 12 pacientes con MAR sin fístula, encontrando una asociación a Síndrome de Down del $50 \% .{ }^{(15)}$

El tratamiento quirúrgico inicial utilizado en la mayoría de los pacientes estudiados 59\% $(n=56)$, comenzó con una colostomía descendente, seguida del abordaje sagital posterior tipo Peña y luego incorporación del paciente al programa de dilataciones anorrectales. El tipo de colostomía mayormente utilizada fue la descendente con dos estomas separados ó colostomía tipo Peña en $74.1 \%(n=60)$.

De los 94 pacientes atendidos en los dos Hospitales Sampedranos durante el período 2012 -2016, únicamente a 33 se les realizó el cierre de la colostomía y 48 aún persisten con la misma; esto está influenciado al estado nutricional, demanda quirúrgica, mala técnica de dilataciones anorrectales.
Se les realizó reparación anorrectal a $84 \%$ $(n=79)$ de los casos; de los cuales $83.1 \%(n=64)$ tuvieron una cirugía exitosa ya que no reportaron complicación alguna y $16.9 \%(n=3)$ presentaron complicaciones siendo la más frecuente estenosis de la anoplastía 3.9\% ( $n=3)$, a diferencia de la literatura internacional donde adjudican que dentro de las complicaciones más frecuentes se encuentra la dehiscencia de la herida quirúrgica e infección. ${ }^{(7,8)}$ El Dr. Alberto Peña afirma que si se repara con la técnica adecuada y de forma meticulosa se puede llegar a un excelente resultado y favorable pronóstico mayor al 50\%. ${ }^{(16)}$

El paciente debe de regresar al establecimiento de salud dos semanas después de haberse sometido al procedimiento quirúrgico de reparación anorrectal, para evitar de esta manera la estenosis de la anoplastía. El ano debe ser dilatado dos veces al día y cada semana aumenta el tamaño del dilatador, el cual depende de la edad del paciente. ${ }^{(1,7)}$ Es importante recalcar el papel fundamental que juegan los padres o encargados de cada paciente con este tipo de malformaciones, ya que asistiendo puntualmente a cada cita médica y cumpliendo con las indicaciones del médico en cuanto a los cuidados de la colostomía y anoplastía así también realizando diariamente y de forma correcta las dilataciones anorrectales en casa para evitar complicaciones futuras. Cabe resaltar, que en algunas situaciones la falta de recursos económicos y/ó el difícil acceso al transporte impide a muchos pacientes asistir puntualmente a todas sus evaluaciones médicas.

Dentro de las limitantes del estudio se encontró que varios expedientes no contaban con historia clínica completa y por este motivo tuvieron que excluirse del presente estudio, otra limitante fue que uno de los Hospitales Sampedranos no cuenta con la cantidad de dilatadores necesarios para poder brindar a 
cada paciente su propio dilatador y de esta manera ofrecer un servicio más eficaz. Es por este motivo que tiene que crearse una política en el Ministerio de Salud para la compra suficiente de los mismos y así tenerlos a disposición de quienes lo necesitan.

En conclusión las malformaciones anorrectales en este estudio afectaron más al sexo femenino que al masculino, la edad que predominó fueron los pacientes de 1 año, procedentes en su mayoría del departamento de Cortés, principalmente del municipio de San Pedro Sula. La fístula rectoperineal fue la malformación anorrectal más frecuente en el sexo masculino, mientras que en el sexo femenino fue la fístula rectovestibular, seguido de la cloaca mayor de $3 \mathrm{cms}$. Menos de un tercio de los pacientes tuvieron asociación a otros defectos congénitos, entre ellos el más común fue hidronefrosis y atresia esofágica. En cuanto al manejo se les realizó la colostomía protectora seguido de Anorrectoplastía Sagital Posterior tipo Peña y por último el programa de dilataciones anorrectales a más de la mitad de los pacientes, con resultados fabulosos ya que la mayoría no presentaron complicaciones.

Se recomienda a la Secretaría de Salud de Honduras crear un programa específicamente para los padres y/o encargados de los pacientes con este tipo de malformaciones congénitas ya que para brindarle una mejor calidad de vida (control de micción e intestinal y función sexual y reproductiva), no solamente es fundamental un examen físico completo y minucioso al momento del nacimiento para poder realizar un diagnóstico temprano, seguido de un tratamiento oportuno con la técnica quirúrgica adecuada a cabo de manos expertas, sino que también la incorporación activa de la familia al tratamiento y al manejo del mismo en forma permanente.
En este estudio realizado en ambos hospitales de San Pedro Sula, se encontró que las malformaciones anorrectales afectaron más a las mujeres $53.2 \%(n=50)$ que a los hombres $46.8 \%$ (44), ligeramente menor a lo reportado en una revisión de cuatro años en Cuba en donde se estudió a 55 recién nacidos intervenidos quirúrgicamente por malformaciones digestivas, atendidos en el Servicio de Neonatología del Hospital Infantil Norte "Dr. Juan de la Cruz Martínez Maceira" de Santiago de Cuba en donde predominaron los varones en un $65.5 \%{ }^{(9)}$

El tipo de malformación anorrectal predominante en el sexo masculino fue la fístula recto-perineal $30.2 \%(n=14)$, y la menos común la fístula recto-cuello-vesical $4.7 \%(n=2)$, a diferencia del sexo femenino en donde predominó la fístula rectovestibular $37.3 \%(n=19)$; similar a los resultado del análisis de 200 casos pediátricos de malformaciones anorrectales en Costa Rica en el Hospital Nacional de Niños "Dr. Carlos Sáenz Herrera" con 92 casos de fístula perineal, 26 fístulas vestibulares y 12 fístulas recto-cueIlo-vesical. ${ }^{(10)}$ Sin embargo, en un estudio retrospectivo de tres años en el que se revisaron 72 expedientes en México por 2 residentes de cirugía pediátrica y 2 cirujanos pediatras en donde los hombres fueron los más afectados con $58.3 \%$ de los casos y la fistula recto uretral Vulvar fue la MAR más común. ${ }^{(11)}$

En el presente estudio hubo otros defectos congénitos en un $29 \%$ ( $n=27$ casos), cifra que difiere a registrado en el archivo de malformaciones congénitas de la maternidad del Hospital Clínico de la Universidad de Chile, en el período comprendido entre 1979 -1999, con una población total de 70,242 nacimientos ocurridos durante el período de estudio, 4,486 con algún tipo de malformación congénita, de los cuales 54 casos tenían algún tipo de malformación anorrectal y el $51.9 \%(n=28)$ de los casos estaban asociados a otra anomalía. ${ }^{(12)}$ 
Otro dato de interés es que en los casos con síndrome de Down $(n=2)$ se asociaron a agenesia anorrectal sin fístula, tal y como lo muestra el estudio retrospectivo de 34 años (19802014) hecho por el Dr. Peña, Bischoff, Frischer y Dickie quienes revisaron los expedientes clínicos de 2,189 pacientes encontrándose con 92 casos de Agenesia Anorrectal sin fístula y con una asociación del $40 \%$ a síndrome de Down. ${ }^{(13)}$ Muy similar al estudio realizado por Fanjul M, Molina E, Cerda J, entre otros en el año 2009 quienes estudiaron 12 pacientes con Malformación Anorrectal sin fístula, en donde encontraron en el $50 \%$ de los mismos $(n=6)$ casos asociados a síndrome de Down. ${ }^{(14)}$

En este estudio, en $59.6 \%(n=56)$ de los pacientes el tratamiento quirúrgico inicial fue la colostomía descendente, seguida del abordaje sagital posterior tipo Peña y luego incorporación del paciente al programa de dilataciones anorrectales; se realizaron al momento del diagnóstico, similar a la revisión de los 200 casos con MAR en Costa Rica en donde se les realizó a 96 pacientes la colostomía protectora, a 72 pacientes la ARPSP y a 96 pacientes la ARPSP limitada. ${ }^{(10)}$

De total de pacientes que entraron en el estudio, se les realizó reparación anorrectal a 79.7\% (75); de los cuales $85.3 \%(n=n=64)$ tuvieron una cirugía exitosa ya que no reportaron complicación alguna con muy buen pronóstico y $14.7 \%$ $(n=11)$ presentaron complicaciones siendo la más frecuente estenosis de la anoplastía 3.9\% $(n=3)$. A diferencia de un estudio de Bailez, en el año 2009, en donde menciona que las complicaciones más frecuentes es la dehiscencia de la herida quirúrgica e infección. ${ }^{(8)}$
Similar a lo reportado en un estudio por Bischoff en el año 2016, un estudio sobre 570 pacientes con cloaca operados por el Dr. Alberto Peña, quien afirma que si se repara con la técnica adecuada y de forma meticulosa se puede llegar a un excelente resultado y favorable pronóstico mayor al 50\%. ${ }^{(15)}$ Estos datos nos demuestran la excelente labor que los cirujanos pediatras de los Hospitales Sampedranos han realizado a lo largo de los años, resolviendo los problemas de salud de los infantes con un mínimo de complicaciones.

En conclusión las Malformaciones Anorrectales en este estudio afectaron más a las mujeres que a los hombres, la edad que predominó fueron los pacientes de 1 año, procedentes en su mayoría del departamento de Cortés, principalmente del municipio de San Pedro Sula. La fístula rectoperineal fue la Malformación Anorrectal más frecuente en el sexo masculino, mientras que en el sexo femenino fue la fístula rectovestibular, seguido de la cloaca mayor de $3 \mathrm{cms}$. Menos de un tercio de los pacientes con Malformaciones Anorrectales tuvieron asociación a otros defectos congénitos, entre ellos el más común fue hidronefrosis y atresia esofágica.

Se recomienda a la Secretaría de Salud de Honduras realizar una Guía de Práctica Clínica para el diagnóstico y tratamiento de las Malformaciones Anorrectales en el período neonatal, con el propósito de optimizar la atención médica brindada a estos pacientes, e incorporarla a nuestro sistema de salud. A los padres de familia y tutores de los pacientes con este tipo de malformación deben ilustrarse respecto a esta temática y seguir las indicaciones médicas en cuanto a las dilataciones anorrectales y el cuidado de la colostomía para obtener mejores resultados. 


\section{BIBLIOGRAFÍA}

1. Peña A. Ano imperforado y malformaciones cloacales. En: Ashcraft, Murphy, Sharp, Sigalet, Snyder. CirugíaPediátrica. 3a. Ed. MacGraw-Hill; 2001. p. 500-520.

2. Alamo L, Meyrat BJ, Meuwly JY, Meuli RA, Gudinchet F. Anorectal malformations: finding the pathway out of the labyrinth. Radiographics. [Internet] 2013 [Citado, 19 marzo 2017]; 33(2): 491-512. Disponible en: https://www.ncbi.nlm.nih.gov/pubmed/ 23479709.

3. Moore KL, Persaud TV, Mark GT. Sistema alimentario. En: Embriología Clínica. 9a. Ed. Madrid: EL SERVIER; 2013. p. 468-527.

4. Holschneider AM, Hutson JM. Anorectal malformations in children: embryology, diagnosis, surgical treatment and followup. Berlin: Springer-Verlag; 2006 p. 2-3.

5. Hernández R, Alvarenga. Frecuencia de malformaciones congénitas externas en recién nacidos de la Unidad Materno Infantil del Hospital Escuela. Factores de riesgo. Rev Med Post UNAH. 2001; 6(2): 148-153.

6. Peña A, Bischoff A. Surgical treatment of colorectal problems in children. Berlin: Springer International; 2015. P. 1-497.

7. Levitt MA, Peña A. Anorectal malformations. Orphanet j RareDis. [Internet] 2007 [Citado, 17 marzo 2017]; 2(33): 1-13. Disponible en: https://ojrd.biomedcentral.com/ articles/10.1186/1750-1172-2-33.

8. Bailez M, Prieto F. Malformaciones Anorrectales. Cirug Diges F. Galindo. [Internet] 2009. [Citado, 21 marzo 2017]; 3(364): 1-19. Disponible en: http://www.sacd.org.ar/tse sentaycuatro.pdf.
9. Garriga M, Joa Mesa T, Dager Haber A, Rubal Wong A. Caracterización de recién nacidos operados a causa de malformaciones digestivas. MEDISAN. [Internet] 2011 [Citado, 21 marzo 2017]; 15(2): 215-221. Disponible en: www.redalyc.org/pdf/3684/ 368445227011.pdf.

10. Ceciliano Romero N, Beauchamp Carvajal D, Chávez Fernández L, Cortés Ojeda J. Análisis de 200 casos pediátricos de malformaciones anorectales. Acta MédCostarric. [Internet] 2010 [Citado, 19 marzo 2017]; 52(2): 109-117. Disponible en: www.scielo. sa.cr/pdf/amc/v52n2/art09v52n2.pdfISSN 0001-6002/2010/52/2/109-117.

11. Baeza Herrera $C$, Rodríguez Calderón $R L$, Villalobos Castillejos A, Martínez Leo BA, Portugal Moreno VH. Ano imperforado. Enfoque clínico-epidemiológico. Acta PediatrMex. Internet] 2013 [Citado, 27 marzo 2017]; 34(2): 55-58 . Disponible en: www.medigraphic.com/pdfs/actpedmex/ apm-2013/apm132b.pd.f

12. Wijers, C.H.W., de Blaauw, I., Marcelis, C.L.M. et al. Research perspectives in the etiology of congenital anorectal malformations using data of the International Consortium on Anorectal Malformations: evidence for risk factors across different populations Pediatr Surg Int (2010) 26: 1093. Disponible en: https://doi.org/10.1007/s00383-010-26 88-0.

13. Nazer J, Hubner G ME, Valenzuela FP, Cifuentes OL. Malformaciones congénitas anorrectales y sus asociaciones preferentes. Experiencia del Hospital Clínico de la Universidad de Chile. Período 1979-1999. Rev. méd. Chile. [Internet] 2000 [Citado, 26 marzo 2017]; 128(5): 519-525. Disponible 
en: http://www.scielo.cl/scielo.php?pid=s 003498872000000500010\&script=sci_art text http://dx.doi.org/10.4067/S0034-9887 2000000500010.

14. Bischoff A, Frischer J, Dickie BH, Peña A. Anorectal malformation without fistula: a defect with unique characteristics. PediatrSurglnt. [Internet] 2014 [Citado, 17 marzo 2017]; 30(8): 763-766. Disponible en: https://www.ncbi.nlm.nih.gov/pubmed/ 24969816 Doi: 10.1007/s00383-014-3527 $-5$.
15. Fanjul M, Molina E, Cerdá J, Parente A, Laín $A$, Cañizo $A$, et al. Characteristics of the anorectal atresia without fistula. Based on 12 cases. [Internet] 2009 [Citado, 18 marzo 2017]; 22(1): 45-8. Disponible en: https:// www.ncbi.nlm.nih.gov/pubmed/193230 83.

16. Bischoff A. The surgical treatment of cloaca. Semin Pediatr Surg. [Internet] 2016 [Citado, 11 marzo 2017]; 25(2): 102-107. Disponible en: https://www.ncbi.nlm.nih.gov/ pubmed/26969234. 